\title{
Dopamine receptor D3R and D4R mRNA levels in peripheral lymphocytes in patients with schizophrenia correlate with severity of illness
} \author{
William G. Honer ${ }^{3}$, Shinji Shimodera ${ }^{1,7}$

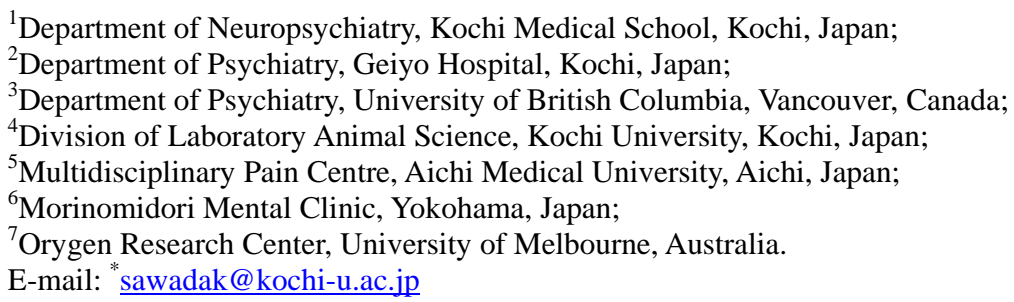

Mitsuhiko Kawano $^{1}$, Ken Sawada ${ }^{1,2,3^{*}}$, Emi Tsuru ${ }^{4}$, Makoto Nishihara ${ }^{5}$, Kunio Kato ${ }^{6}$,

Received 16 May 2011; revised 20 June 2011; accepted 29 June 2011.

\begin{abstract}
Schizophrenia is a disease that affects many areas of the brain. The dopamine hypothesis is one of the most widely-accepted ideas in the pathophysiology of schizophrenia. Besides alterations in the dopaminergic system in the central nervous system, there have been several reports of changes in dopaminergic systems in the peripheral blood of schizophrenic patients. Several reports have shown that dopamine receptor expression by lymphocytes is altered in patients with schizophrenia, but the results have been conflicting. We therefore re-assessed D3R and D4R mRNA levels in 11 patients with schizophrenia and 12 healthy subjects and correlated levels with severity of symptoms. D3R and D4R expression in lymphocytes and granulocytes was measured by quantitative RT-PCR and the severity of symptoms and cognitive impairment were assessed using the PANSS and BACS-J. There were no significant differences in mean D3R or D4R mRNA levels in lymphocytes from schizophrenic patients and controls and no significant difference in mean D4R mRNA levels in granulocytes (D3R mRNA undetectable). In patients with schizophrenia, D3R expression was inversely correlated with the total PANSS score $(r=0.768, p=0.009)$, while D4R expression was positively correlated with working memory scales $(r=0.895, p=0.001)$. In conclusion, these results imply that lymphocyte D3R and D4R are involved in the mechanisms of the disorder and could be used as target markers in the treatment
\end{abstract}

of schizophrenia.

Keywords: Dopamine receptor D3R; Dopamine receptor D4R; Schizophrenia; RT-PCR; Lymphocyte; Cognitive function

\section{INTRODUCTION}

Schizophrenia is a disease that affects diverse brain regions, causing patients to show a variety of clinical manifestations, such as psychopathologies and cogni- tive deficits [1]. Intensive study for more than 30 years has led to the proposal that alteration of dopaminergic neurotransmission is implicated in the pathophysiology of schizophrenia [2,3]. The dopamine hypothesis of schizophrenia postulates that excessive dopaminergic neurotransmission in the limbic cortex is responsible for positive symptoms, such as hallucinations and delusions, while limited dopaminergic activity in the frontal cortex results in negative symptoms, such as avolition and anhednia, and in cognitive dysfunction [4].

Dopamine, a major neurotransmitter in the central nervous system (CNS), regulates motor coordination, cognition, mood, and reward, and acts on the endocrine and cardiovascular systems. The dopamine receptor family consists of five seven-transmembrane domain $G$ protein-coupled receptors termed D1R to D5R [5,6], which are classified into the two groups of D1-like receptors (D1R and D5R) and D2-like receptors (D2R, D3R, and D4R). D2-like receptors have attracted pharmacological interest for the design of antipsychotics, 
because the therapeutic benefits of these medications are reported to be associated with their antagonistic action on D2-like receptors in the brain. D3R is highly expressed in areas of the limbic cortex, such as the nucleus accumbens and islands of Calleja, and moderately expressed in the striatum and dentate gyrus [7-9], while D4R mRNA is expressed in the prefrontal and temporal cortex and hippocampus [8,10-13]. A postmortem study showed that D3 mRNA levels are selectively decreased in the parietal cortex and motor cortex of schizophrenic patients [14], and a focal decrease in D3R and D4R mRNA levels in the orbitofrontal cortex has been reported [11].

Several studies have shown that dopamine receptor mRNA and protein levels are correlated with the symptoms of schizophrenia. D3 polymorphism was found to be associated with improvement of symptoms after 10 weeks' treatment in first-episode drug-naïve schizophrenia [15]. Polymorphism of D4R was correlated with working memory in a near-infrared spectroscopy study [16], while an animal study suggested an association between D4R and working memory [17].

The CNS and peripheral nervous system modulate peripheral immune cells by releasing neuropeptides and neurotransmitters [18]. Lymphocytes themselves produce dopamine [19], and the expression of dopamine receptors on lymphocytes has been confirmed by quantification of mRNA [20]. Dopamine is an important regulator of T lymphocyte functions [21]. RT-PCR and immunostaining studies on lymphocytes showed no detectable D2 mRNA, but detectable D3R and D4R mRNA [22-24]. D3R and D4R mRNA levels in lymphocytes are comparable to, but D2R and DR5R mRNA levels are significantly lower than, those in the brain [20]. Several studies have examined D3R or D4R mRNA levels in lymphocytes and $\mathrm{T}$ cells in schizophrenia, but the results have been conflicting. D3R mRNA levels have been reported to be elevated in lymphocytes from schizophrenic patients, while D4R mRNA levels were found to be unaltered [25]. D3R mRNA levels in lymphocytes from drug naïve schizophrenia were found to be increased compared to controls [26]. Another report found significantly higher levels of D3R mRNA in T cells from schizophrenics, and a significant reduction in D4R mRNA levels in CD4 + T cells [27], while two other papers reported no alteration in D3R mRNA levels in peripheral blood in the acute first episode of schizophrenia [28] or no difference in D3R and D4R protein levels in peripheral leukocytes between schizophrenics and controls [29]. Thus, it is not clear whether dopamine receptor mRNA levels are altered in the peripheral blood of patients with schizophrenia.

To validate the use of D3R and D4R as peripheral markers in schizophrenia, we reexamined whether there was a change in dopamine receptor expression using the real-time reverse transcriptase-polymerase chain reaction (RT-PCR) to quantify levels of D3R and D4R mRNAs in lymphocytes and granulocytes in the peripheral blood of patients with schizophrenia. Furthermore, we investigated correlations between dopamine receptor expression levels and clinical manifestations or scores in mental state examination and cognitive function assessments. We used the following hypotheses to plan this study.

1) In terms of mRNA expression in the peripheral blood, D3R is increased and D4R decreased in patients with schizophrenia compared to the control group.

2) The alteration in D3R and D4R levels is related to the severity of the clinical manifestations and cognitive dysfunction in patients with schizophrenia.

\section{METHODS}

\subsection{Participants}

Eleven patients (age $56 \pm 12$ years (mean \pm SD), 8 males) with schizophrenia diagnosed using DSM-IV and twelve healthy controls ( $44 \pm 12$ years, 3 males) were enrolled in this study. All of the participants provided written informed consent for participation in this study. The patients with schizophrenia were evaluated using the Positive and Negative Syndrome Scale (PANSS) and the Brief Assessment of Cognition in Schizophrenia (BACS-J), which are composed of Verbal Memory, Digit Sequencing, Token Motor Task, Verbal Fluency, Symbol Coding task, and Tower of London. The patients were treated with aripiprazole $(n=4)$, olanzapine $(n=2)$, perospirone $(n=2)$, haloperidol $(n=2)$, or fluphenazine decanoate $(n=1)$. The doses of antipsychotics were calculated as chlorpromazine-equivalent doses. The study was approved by the Ethics Committee of Kochi Medical School.

\subsection{Sample Processing}

Peripheral blood, collected between 10:00 a.m. and noon, was layered on Monopoly Separation Solution (DS Pharma Biomedical) and separated into lymphocytes and granulocytes according to the manufacture's instructions.

\subsection{RT-PCR}

For RT-PCR analysis, total RNA was isolated using TRIzol (Invitrogen). Total RNA $(0.5 \mu \mathrm{g})$ was reverse transcribed using Primescript reverse transcriptase (Ta$\mathrm{KaRa}$ ) and random 6-mers and oligo dT primer (TaKaRa) according to the manufacturer's instructions. The resulting complementary DNA fragments were amplified using Fast Start Universal Probe Master Mix (ROX reference dye) (Roche) according to the manufacturer's in structions. Reactions were run using an ABI PRISM 
7000 Sequence Detection System (Applied Biosystems) under the following conditions: $50^{\circ} \mathrm{C}$ for $2 \mathrm{~min}, 95^{\circ} \mathrm{C}$ for $10 \mathrm{~min}$, followed by 42 cycles of $95^{\circ} \mathrm{C}$ for $15 \mathrm{~s}$ and $60^{\circ} \mathrm{C}$ for $1 \mathrm{~min}$. Primers and probes were designed using the Universal Probe Library Assay program (https://www. roche-applied-science.com/sis/rtpcr/upl/in dex.jsp). The primers and the probe for $\beta$-actin mRNA was purchased from Roche. The RT-PCR data were normalized to those for the housekeeping gene $\beta$-actin. Relative mRNA expression was calculated using the delta-Ct-method.

\subsection{Statistical analysis}

All statistical calculations were performed using the SPSS statistical package version 11.0. To examine the difference in age and sex between the groups, the chisquare analysis was used for sex and the Mann-Whitney test for age. We performed the Multivariate Generalized Linear Models to evaluate whether age and sex affected dopamine receptor mRNA levels. To compare differences in D3R and D4R mRNA levels between patients and controls, we used the Mann-Whitney test. To exclude the influence of possible confounders on dopamine receptor mRNA levels, we performed the Spearman test to determine whether there was a correlation between mRNA levels and age, dose of antipsychotics, or duration of the illness. Furthermore, because of the non-continuous nature of the PANSS and BACS-J data, we analyzed correlations between D3R and D4R mRNA levels and the PANSS and subscales of the BACS-J using the Spearman test. A Bonferroni-correction was applied to correct for multiple comparisons. We considered statistical significant in multiple comparisons as $p<0.013$ (comparing granulocyte D4R mRNA and lymphocyte D3R and D4R mRNA levels, 3 comparisons) or $p<0.00278$ (comparing D3R and D4R mRNA levels and subscales of BACS-J, 18 comparisons).

\section{RESULTS}

The average duration of illness in the patient group was 25.2 years. The patients had a total PANSS score of 83.6 \pm 13.8 (Table 1). The control group and patient group had a different mean age and percentage of each sex (age: $p=0.028$, sex: $p=0.041$ ). However, D3R and D4R mRNA levels in lymphocytes or D4R mRNA levels (D3R mRNA levels were undetectable) in granulocytes measured by real-time RT-PCR were not influenced by age or sex (age: Wilks's lambda $F=3.95, d f=4,27, p=$ 0.22 , sex: Wilks's lambda $F=42.57, d f=4,13, p=$ $0.11)$.

D2R, D3R, and D4R mRNA levels were measured in granulocytes and lymphocytes, but D2R mRNA levels in lymphocytes and granulocytes and D3R mRNA levels in Table 1. Demographic and clinical characteristics of the patients and normal controls.

\begin{tabular}{lcc}
\hline & Schizophrenia & Control \\
\hline Sex (M:F) & $8: 3$ & $3: 9$ \\
Age & $54.2 \pm 10.7$ & $43.8 \pm 12.0$ \\
Duration of illness & $25.2 \pm 15.3(n=11)$ & \\
PANSS total & $83.6 \pm 13.8$ & \\
BACS-verbal memory & $-1.55 \pm 0.96$ & \\
BACS-working memory & $-1.24 \pm 1.29$ & \\
BACS-motor speed & $-3.87 \pm 2.87(n=10)$ & \\
BACS-verbal fluency & $-1.23 \pm 1.75$ & \\
BACS-attention & $-3.14 \pm 1.91$ & \\
BACS-executive & $-1.59 \pm 2.04$ & \\
BACS-composite & $-2.10 \pm 1.45$ & \\
Lymphocyte D3 & $0.52 \pm 0.26(n=9)$ & $0.63 \pm 0.27$ \\
Lymphocyte D4 & $0.49 \pm 0.09$ & $0.51 \pm 0.11$ \\
Granulocyte D4 & $0.44 \pm 0.18$ & $0.44 \pm 0.12$ \\
\hline
\end{tabular}

granulocytes were very low and we therefore did not analyze these further. The analysis showed that there was no statistically significant difference between patients and controls in terms of lymphocyte D3R levels ( $p$ $=0.286)$, lymphocyte D4R levels ( $p=0.477)$, or granulocyte D4R levels ( $p=0.792$ ) (Figure 1).

To exclude the effects of antipsychotics and duration of the illness, we analyzed correlations between receptor mRNA levels and dose of antipsychotics or duration of illness, and found no statistically significant correlation with lymphocyte D3R mRNA levels (antipsychotics: $p=$ 0.795, duration: $p=0.100$ ), lymphocyte D4R mRNA levels (antipsychotics: $p=0.985$, duration: $p=0.464$ ) or granulocyte D4R mRNA levels (antipsychotics: $p=$ 0.253 , duration: $p=0.136$ ). Lymphocyte D3R mRNA or lymphocyte or granulocyte D4R mRNA levels were also not significantly correlated with duration of the illness.

Next, to examine the relevance of dopamine receptor mRNA levels to clinical factors, we examined correlations between dopamine receptor mRNA levels and the PANSS and BACS-J scores. Although the correlation between lymphocyte D3R mRNA levels and the PANSS subscales did not reach statistical significance, lymphocyte D3R mRNA levels and total PANSS score showed a statistically significant inverse relationship (Spearman, $r$ $=0.768, p=0.009$ ). Lymphocyte D4R mRNA levels and the score on the working memory subscale of the BACS-J also showed a strong positive correlation (Spearman, $r=0.895, p=0.001$ ) (Figure 2).

\section{DISCUSSION}


Although we successfully quantified levels of mRNAs Lymphocyte D3 Lymphocyte D4 Granulocyte D4
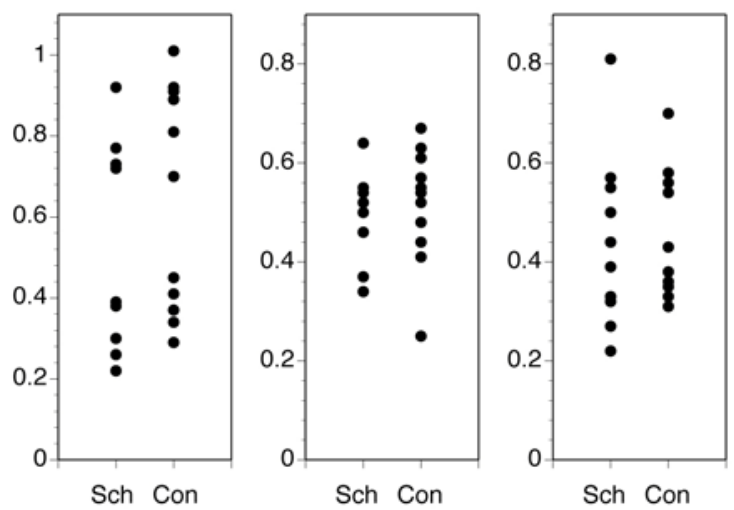

Figure 1. D3R and D4R mRNA levels in lymphocytes and D4R mRNA levels in granulocytes. The y-axis shows the relative expression level. Sch: schizophrenia, Con: Control

for lymphocyte D3R and D4R and granulocyte D4R, there were no statistically significant differences in the levels of these receptor mRNAs in patients with schizophrenia and controls. Consequently, this study failed to replicate the previous results of either elevated D3R mRNA levels [25] or reduced D4R levels in lymphocytes [27]. However, among the patients with schizophrenia, lymphocyte D3R mRNA levels showed a negative correlation with the total PANSS score. In addition, there was a strong correlation between lymphocyte D4R mRNA levels and the score for working memory.

Several studies have indicated that D3R mRNA levels correlate with the clinical assessment. A study showed a negative correlation between D3R mRNA levels in lymphocytes and the personality trait of persistence in the normal population [28]. The Ser9Gly polymorphism of D3R is associated with severity of symptoms of the first-episode of schizophrenia in drug-naive patients [15]. Lower levels of lymphocyte D3R protein in schizophrenia correlated with a higher hebephrenic subscale score of the BPRS [29]. Consistent with this last report, our results showed that the severity of psychotic symptoms in patients with schizophrenia correlated with lower lymphocyte D3R mRNA levels. However, our results conflict with the increase in lymphocyte D3R mRNA levels reported in patients with schizophrenia, taken as a whole, compared to healthy controls [25]. As the patients we recruited were relatively chronic and showed higher symptom scores, it is possible that D3R mRNA levels may have been reduced.

In addition to the correlation with D3R, this study demonstrated that lymphocyte D4R mRNA levels showed a strong positive correlation with the scale of working memory, a subscale on the BACS-J.
Many studies have demonstrated that D4R levels have

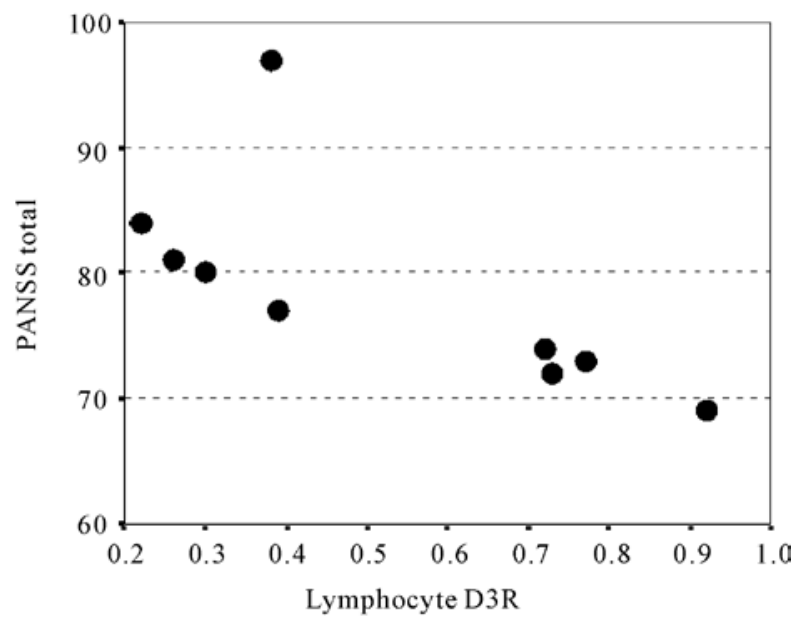

(a)

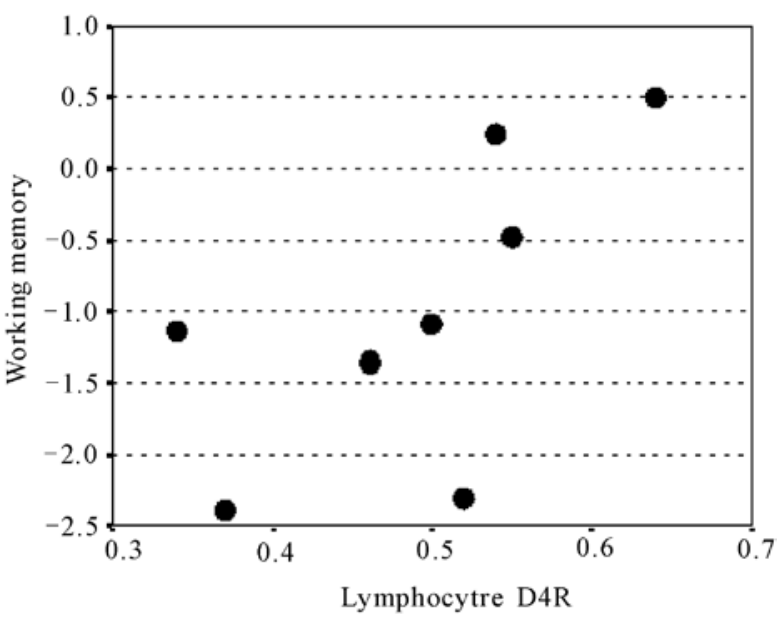

(b)

Figure 2. (a) Correlation plot of the total PANSS score versus lymphocyte D3R mRNA levels; (b) Correlation plot of the working memory score on the BACS-J versus lymphocyte D4R mRNA levels.

an important implication for working memory. A functional imaging study showed that variation in the number of allele repeats in D4R exon II is associated with working memory [16]. During working memory, gamma aminobutyric acid GABAergic interneurons control the activity of pyramidal neurons in the prefrontal cortex [30] and disturbance of GABAergic inhibition results in impairment of working memory [31]. Recently, a mechanism in which the D4R is involved in working memory was proposed. Electrophysiological and immunofluorescence studies have shown that $\mathrm{D} 4 \mathrm{R}$ activation leads to depression of actin-based trafficking of $\alpha$-amino -3-hydroxy-5-methyl-4-isoxazolepropionic acid receptors and excitatory transmission in the GABAergic interneurons of the prefrontal cortex [32] and D4R activa- 
tion controls the myosin-based transport of the GABAA receptor in the prefrontal cortex [33]. Conversely, a reduction in the D4R in the cerebral cortex (prefrontal cortex) might worsen working memory and the reduction might result in a decrease in lymphocyte D4R levels. Since we did not perform the BACS-J on the control group, it is not known whether these correlations also apply to the normal population.

Previous studies have shown that lymphocytes in the blood interact with the CNS through the blood-brain barrier (BBB). In neurodegenerative disorders, such as multiple sclerosis and Parkinson's disease, lymphocytes can cross the BBB to act on the CNS [34,35]. A microarray study showed that, of the 45 candidate genes relevant to schizophrenia, 21 are expressed in both whole blood and prefrontal cortex. Furthermore, the level of expression of many genes, including those for which the protein product is involved in exocytosis and neurogenesis, is not significantly different between whole blood and prefrontal cortex [36]. Thus, the peripheral alterations in schizophrenia possibly reflect impairment in the CNS. The availability of an accessible tissue, such as the blood, with an mRNA expression profile similar to that of the CNS has the potential to advance research in schizophrenia.

One confounding factor in this study was the long-term treatment of the patients. Although it was possible that antipsychotics might affect dopamine receptors in the peripheral blood, our results showed no correlation between antipsychotic medication and D3R or D4R mRNA levels. A previous report also showed that the type of antipsychotic does not affect D3R and D4R mRNA levels [25]. Another study showed that patients treated with olanzapine for 16 days show no increase in D3R mRNA levels [37]. In addition, duration of illness was not correlated with dopamine receptor expression in our study. To clearly determine whether antipsychotics change the expression of dopamine receptors, animal experiments using antipsychotics and psychomimetics are required.

There are several limitations to this study. Firstly, the sample population was small and a large-scale study is required to confirm the correlations. Secondly, we measured mRNA levels, and not amounts of protein. The levels and functions of D3R and D4R protein in peripheral blood are not known, Flow cytometry would be useful for double-staining studies on peripheral blood. Thirdly, the lymphocyte population used consisted of B lymphocytes, natural killer cells, and T lymphocytes and the next stage would be to separate these cell subtypes and investigate D3R and D4R expression. Despite these limitations, this study provides a preliminary insight into the function of D3R and D4R in peripheral blood in pa- tients with schizophrenia, which may have diagnostic importance for evaluating the severity of symptoms of and cognitive function.

In conclusion, this study found that D3R and D4R mRNA levels in the peripheral blood in patients with schizophrenia were unchanged compared to controls. However, patients with a higher total score on the PANSS had lower levels of lymphocyte D3R mRNA and patients with a higher score in the digit sequencing task (better working memory) on the BACS-J had higher lymphocyte D4R mRNA levels. D3R and D4R mRNA in peripheral lymphocyte can therefore be used as a measure of severity of schizophrenia. We also need to evaluate an appropriate cognitive function in a control group to examine whether there is also a correlation with D4R mRNA levels in the normal population. Measurement of protein levels involving a large number of cases are needed to confirm our results. Further work to replicate and validate the correlation between lymphocyte D3R and D4R levels and clinical manifestations should provide useful information for the assessment and management of schizophrenia.

\section{ACKNOWLEDGEMENTS}

This research was partially supported by the Ministry of Education, Science, Sports and Culture, Grant-in-Aid for 19591359 and Kochi Medical School Alumni Association.

We thank Dr. Kaneda who kindly provided suggestions for analyzing cognitive function.

\section{REFERENCES}

[1] Schultz, S.K. and Andreasen, N.C. (1999) Schizophrenia. Lancet, 353, 1425-1430.

doi:10.1016/S0140-6736(98)07549-7

[2] Seeman, P. (1987) Dopamine receptors and the dopamine hypothesis of schizophrenia. Synapse, 1, 133-52. doi:10.1002/syn.890010203

[3] Howes, O.D. and Kapur, S. (2009) The dopamine hypothesis of schizophrenia: Version III-the final common pathway. Schizophr Bull, 35, 549-562. doi:10.1093/schbul/sbp006

[4] Kerwin, R. (2000) From pharmacological profiles to clinical outcomes. International Clinical Psychopharmacology, 15, S1-S4.

[5] Missale, C., Nash, S.R., Robinson, S.W., et al. (1998) Dopamine receptors: From structure to function. Physiological Review, 78, 189-225.

[6] Sibley, D.R., Monsma, F.J.Jr. and Shen, Y. (1993) Molecular neurobiology of dopaminergic receptors. International Review of Neurobiology, 35, 391-415.

doi:10.1016/S0074-7742(08)60573-5

[7] Landwehrmeyer, B., Mengod, G. and Palacios, J.M. (1993) Dopamine D3 receptor mRNA and binding sites in human brain. Molecular Brain Research, 18, 187-92. doi:10.1016/0169-328X(93)90188-U

[8] Meador-Woodruff, J.H., Damask, S.P., Wang, J., et al. 
(1996) Dopamine receptor mRNA expression in human striatum and neocortex. Neuropsychopharmacology, 15, 17-29. doi:10.1016/0893-133X(95)00150-C

[9] Suzuki, M., Hurd, Y.L., Sokoloff, P., et al. (1998) $\mathrm{D}_{3}$ dopamine receptor mRNA is widely expressed in the human brain. Brain Research, 779, 58-74. doi:10.1016/S0006-8993(97)01078-0

[10] Meador-Woodruff, J.H., Grandy, D.K., Van Tol, H.H., et al. (1994) Dopamine receptor gene expression in the human medial temporal lobe. Neuropsychopharmacology, 10, 239-248.

[11] Meador-Woodruff, J.H., Haroutunian, V., Powchik, P., et al. (1997) Dopamine receptor transcript expression in striatum and prefrontal and occipital cortex. Focal abnormalities in orbitofrontal cortex in schizophrenia. Archives of General Psychiatry, 54, 1089-1095.

[12] Matsumoto, M., Hidaka, K., Tada, S., et al. (1996) Low levels of mRNA for dopamine D4 receptor in human cerebral cortex and striatum. Journal of Neurochemistry, 66, 915-919. doi:10.1046/j.1471-4159.1996.66030915.x

[13] Mulcrone, J. and Kerwin, R.W. (1996) No difference in the expression of the D4 gene in post-mortem frontal cortex from controls and schizophrenics. Neuroscience Letters, 219, 163-166. doi:10.1016/S0304-3940(96)13191-8

[14] Schmauss, C., Haroutunian, V., Davis, K.L., et al. (1993) Selective loss of dopamine $\mathrm{D}_{3}$-type receptor mRNA expression in parietal and motor cortices of patients with chronic schizophrenia. Proceeding of National Academy Science of United States of America, 90, 8942-8946. doi:10.1073/pnas.90.19.8942

[15] Reynolds, G.P., Yao, Z., Zhang, X., et al. (2005) Pharmacogenetics of treatment in first-episode schizophrenia: D3 and 5-HT2C receptor polymorphisms separately associate with positive and negative symptom response. European Neuropsychopharmacology, 15, 143-151. doi:10.1016/j.euroneuro.2004.07.001

[16] Herrmann, M.J., Walter, A., Schreppel, T., et al. (2007) $\mathrm{D}_{4}$ receptor gene variation modulates activation of prefrontal cortex during working memory. Neuroscience, 26, 2713-2718. doi:10.1111/j.1460-9568.2007.05921.x

[17] Zhang, K., Grady, C.J., Tsapakis, E.M., et al. (2004) Regulation of working memory by dopamine D4 receptor in rats. Neuropsychopharmacology, 29, 1648-1655. doi:10.1038/sj.npp.1300491

[18] Qiu, Y., Peng, Y. and Wang, J. (1996) Immunoregulatory role of neurotransmitters. Advance in Neuroimmunology, 6, 223-231. doi:10.1016/S0960-5428(96)00018-6

[19] Josefsson, E., Bergquist, J., Ekman, R., et al. (1996) Catecholamines are synthesized by mouse lymphocytes and regulate function of these cells by induction of apoptosis. Immunology, 88, 140-146. doi:10.1046/j.1365-2567.1996.d01-653.x

[20] Kirillova, G.P., Hrutkay, R.J., Shurin, M.R., et al. (2008) Dopamine receptors in human lymphocytes: Radioligand binding and quantitative RT-PCR assays. Journal of Neuroscience Methods, 174, 272-280. doi:10.1016/j.jneumeth.2008.07.018

[21] Sarkar, C., Das, S., Chakroborty, D., et al. (2006) Cutting Edge: Stimulation of dopamine $\mathrm{D}_{4}$ receptors induce $\mathrm{T}$ cell quiescence by up-regulating Kruppel-like factor-2 expression through inhibition of ERK1/ERK2 phospho- rylation. Journal of Immunology, 177, 7525-7529.

[22] Vile, J.M. and Strange, P.G. (1996) $\mathrm{D}_{2}$-like dopamine receptors are not detectable on human peripheral blood lymphocytes. Official Journal of the Society of Biological Psychiatry, 40, 881-885. doi:10.1016/0006-3223(95)00498-X

[23] Amenta, F., Bronzetti, E., Felici, L., et al. (1999) Dopamine D2-like receptors on human peripheral blood lymphocytes: A radioligand binding assay and immunocytochemical study. Autonomic and Autacoid Pharmacology, 19, 151-159. doi:10.1046/j.1365-2680.1999.00135.x

[24] Ricci, A., Bronzetti, E., Felici, L., et al. (1998) Labeling of dopamine D3 and D4 receptor subtypes in human peripheral blood lymphocytes with [3H]7-OH-DPAT: A combined radioligand binding assay and immunochemical study. Journal of Neuroimmunology, 92, 191-195. doi:10.1016/S0165-5728(98)00207-0

[25] Ilani, T., Ben-Shachar, D., Strous, R.D., et al. (2001) A peripheral marker for schizophrenia: Increased levels of $\mathrm{D}_{3}$ dopamine receptor mRNA in blood lymphocytes. Proceeding of National Academy Science of the United States of America, 98, 625-628. doi:10.1073/pnas.021535398

[26] Kwak, Y.T., Koo, M.S., Choi, C.H., et al. (2001) Change of dopamine receptor mRNA expression in lymphocyte of schizophrenic patients. BMC Medical Genetics, 2, 3. doi:10.1186/1471-2350-2-3

[27] Boneberg, E.M., von Seydlitz, E., Propster, K., et al. (2006) $D_{3}$ dopamine receptor mRNA is elevated in $T$ cells of schizophrenic patients whereas $\mathrm{D}_{4}$ dopamine receptor mRNA is reduced in CD4+ -T cells. Journal of Neuroimmunology, 173, 180-187. doi:10.1016/j.jneuroim.2005.11.018

[28] Czermak, C., Lehofer, M., Renger, H., et al. (2004) Dopamine receptor $D_{3}$ mRNA expression in human lymphocytes is negatively correlated with the personality trait of persistence. Journal of Neuroimmunology, 150, 145-149. doi:10.1016/j.jneuroim.2004.01.009

[29] Rodrigues, K.P., Souza, P.A., Lima, P.M., et al. (2005) Expression of $\mathrm{D}_{3}$ and $\mathrm{D}_{4}$ dopamine receptors in leukocytes is related to schizophrenic symptoms. Schizophrenia Research, 80, 363-365. doi:10.1016/j.schres.2005.06.010

[30] Constantinidis, C., Williams, G.V. and Goldman-Rakic, P.S. (2002) A role for inhibition in shaping the temporal flow of information in prefrontal cortex. Nature Neuroscience, 5, 175-180. doi:10.1038/nn799

[31] Lewis, D.A., Hashimoto, T. and Volk, D.W. (2005) Cortical inhibitory neurons and schizophrenia. Nature Review Neuroscience, 6, 312-324. doi:10.1038/nrn1648

[32] Yuen, E.Y. and Yan, Z. (2009) Dopamine $\mathrm{D}_{4}$ receptors regulate AMPA receptor trafficking and glutamatergic transmission in GABAergic interneurons of prefrontal cortex. Journal of Neuroscience, 29, 550-562. doi:10.1523/JNEUROSCI.5050-08.2009

[33] Graziane, N.M., Yuen, E.Y. and Yan, Z. (2009) Dopamine $D_{4}$ receptors regulate GABAA receptor trafficking via an actin/cofilin/myosin-dependent mechanism. Journal of Biological Chemistry, 284, 8329-8336. doi:10.1074/jbc.M807387200

[34] Engelhardt, B. (2008) Immune cell entry into the central nervous system: Involvement of adhesion molecules and 
chemokines. Journal of Neurological Science, 274, 23-26. doi:10.1016/j.jns.2008.05.019

[35] Brochard, V., Combadiere, B., Prigent, A., et al. (2009) Infiltration of $\mathrm{CD}_{4}+$ lymphocytes into the brain contributes to neurodegeneration in a mouse model of Parkinson disease. Journal of Clinical Investigation, 119, 182-192.

doi:10.1172/JCI36470

[36] Sullivan, P.F., Fan, C. and Perou, C.M. (2006) Evaluating the comparability of gene expression in blood and brain American Journal of Medical Genetics, 141B, 261-268. doi:10.1002/ajmg.b.30272

[37] Padin, J.F., Rodriguez, M.A., Dominguez, E., et al. (2006) Parallel regulation by olanzapine of the patterns of expression of 5-HT2A and $\mathrm{D}_{3}$ receptors in rat central nervous system and blood cells. Neuropharmacology, 51, 923-932. doi:10.1016/j.neuropharm.2006.06.005 